\title{
Cognitive and Mood Functioning in Borderline and Schizotypal Personality Disorders
}

\author{
Kim E. Goldstein', Heather A. Berlin', Holly K. Hamilton1, Effie M. Mitsis ${ }^{1,2}$, \\ Margaret M. McClure', Kimberley R. Savage ${ }^{1}$, Nicholas J. Blair ${ }^{1,3}$, Michelle R. Feder ${ }^{1,3}$, \\ Larry J. Siever ${ }^{1,3}$, Antonia S. New ${ }^{1}$, Erin A. Hazlett 1,3,4* \\ ${ }^{1}$ Department of Psychiatry, Icahn School of Medicine at Mount Sinai, New York, NY, USA \\ ${ }^{2}$ Department of Rehabilitation Medicine, James J. Peters VA Medical Center, Bronx, NY, USA \\ ${ }^{3}$ Mental IIIness Research, Education and Clinical Center (VISN3 MIRECC), James J. Peters VA Medical Center, \\ Bronx, NY, USA \\ ${ }^{4}$ Department of Neuroscience, Icahn School of Medicine at Mount Sinai, New York, NY, USA \\ Email: *erin.hazlett@mssm.edu
}

Received 19 December 2015; accepted 13 March 2016; published 16 March 2016

Copyright @ 2016 by authors and Scientific Research Publishing Inc.

This work is licensed under the Creative Commons Attribution International License (CC BY).

http://creativecommons.org/licenses/by/4.0/

(c) (i) Open Access

\section{Abstract}

Research suggests many shared clinical features across individuals with Schizotypal Personality Disorder (SPD) and Borderline Personality Disorder (BPD), including problems with attention/ executive functioning and mood. Therefore, aspects of these areas of functioning were compared in SPD and BPD to better characterize their respective difficulties. BPD, SPD, and healthy control (HC) participants were administered measures of cognitive and mood functioning. Compared with healthy controls, SPD patients performed significantly worse on aspects of the Delayed-Matchingto-Sample task, a measure of short-term visual memory abilities; however, the individuals with BPD did not differ from healthy controls. Neither of the patient groups differed from HC's on measures of processing speed or planning. With regard to mood functioning, the BPD group exhibited significantly higher levels of affective disturbance (e.g., sadness, fear, anger) compared with the SPD patients and HCs. Overall, findings suggest different patterns of fronto-subcortical weakness in each patient group. While SPD patients exhibited relative weakness with short-term memory, BPD patient performance on such measures did not reveal relative weakness compared with HCs but did implicate problems with mood.

\section{Keywords}

Neuropsychology, Neurocognition, Short-Term Visual Memory, Emotion, CANTAB, Personality

*Corresponding author.

How to cite this paper: Goldstein, K. E., Berlin, H. A., Hamilton, H. K., Mitsis, E. M., McClure, M. M., Savage, K. R., Blair, N. J., Feder, M. R., Siever, L. J., New, A. S., \& Hazlett, E. A. (2016). Cognitive and Mood Functioning in Borderline and Schizotypal Personality Disorders. Psychology, 7, 292-299. http://dx.doi.org/10.4236/psych.2016.73032 


\section{Disorder}

\section{Introduction}

Personality disorders are pervasive in the general population, co-occur with major mental disorders, and are associated with serious impairment (Lenzenweger et al., 2007). Of the ten personality disorders, Schizotypal Personality Disorder (SPD) and Borderline Personality Disorder BPD) are two of the most empirically studied. SPD is characterized by asocial tendencies, difficulties with language, paranoid, odd behavior, and magical thinking. $\mathrm{BPD}$, on the other hand, is characterized by affective instability, impulsive behavior, and disrupted interpersonal relationships and self-image.

Nonetheless, research suggests many shared clinical features across these two personality disorders, such as cognitive/perceptual dysfunction and paranoia (McGlashan, 1987; Kavoussi \& Siever, 1992), similar biological markers, such as frontal and temporal lobe abnormalities (McCloskey et al., 2005), and comparable neuropsychological difficulties, including problems with aspects of attention/executive functioning (Seres, Unoka, Nikoletta, Áspán, \& Keri, 2009). For example, previous studies reviewing neuropsychological weaknesses in individuals with BPD document disruptions in flexibility, working memory, decision-making, and inhibition (LeGris \& Reekum, 2006; Dell'Osso et al., 2010). Prior work examining neuropsychological functioning in SPD individuals primarily implicates problems with attention, processing speed, and working memory (Mitropoulou et al., 2002, 2005; Ruocco, 2005). Still, despite overlapping areas of neuropsychological weakness, BPD and SPD groups have been shown to differ phenomenologically in some areas: emotion dysregulation and self-damaging acts characterize BPD individuals, whereas blunted affect and social isolation frequently characterize SPD (McGlashan, 1987). Given these potentially overlapping and diverging neurobiological and phenotypic aspects of BPD and SPD, as well as the inherent complexity of both, this study offers a unique opportunity for clarifying neuropsychological and mood commonalities/distinctions in two separate personality disorders.

The primary goal of the current study was to elucidate aspects of neuropsychological and mood functioning in BPD and SPD, respectively, by investigating areas where these two personality disorder groups exhibit potential weakness, particularly within the domains implicated in prior work conducted by our group and others (e.g., attention/executive functioning, mood). Consistent with previous work, we hypothesized that BPD individuals would demonstrate reduced planning and working memory abilities, whereas SPD individuals would exhibit primary difficulties with attention, processing speed, and working memory. Furthermore, we hypothesized that individuals with BPD would report heightened levels of affective disturbance, including significantly higher levels of sadness, anger, and fear, compared with SPD patients and healthy controls.

\section{Methods}

\subsection{Participants}

Fifty-three adults participated in the study: 18 with BPD but no SPD traits, 18 with SPD but no BPD traits, and 17 HCs. Participants in the three groups did not differ on age, gender, education, or performance on a brief measure of general cognitive functioning (See Table 1), which allowed us to match the participants on these factors. We have previously published neuropsychological performance data on the Spatial Working Memory (SWM) subtest of the Cambridge Neuropsychological Test Automated Battery (CANTAB) from a subset of the sample described in this study in the context of a structural MRI study (Goldstein et al., 2011).

All participants were diagnosed using the Structured Clinical Interview for DSM-IV Axis I disorders (SCID-I; First, Spitzer, Gibbon, \& Williams, 1996) and the Structured Interview for DSM-IV Personality Disorders (SIDP-IV; Pfohl, Blum, \& Zimmerman, 1997). Participants either met no Axis I or II DSM-IV criteria (HCs), or met DSM-IV criteria for BPD or SPD. Control participants with a first-degree relative with an Axis I disorder (as assessed via clinical interview by questions posed to the participant) were excluded from the study. BPD and SPD participants had no lifetime history of bipolar (type I) disorder, schizophrenia, a psychotic disorder, or unipolar affective disorder and had not taken any psychoactive medication in the last two weeks. Participants were excluded if they had severe medical or neurological illness (i.e., severe pathological condition of the brain/body, including any aspects of the nervous system), past substance dependence, or substance abuse within the past six 
Table 1. Demographics of the borderline personality disorder, schizotypal personality disorder, and healthy control samples. Standard deviations are provided in parentheses. WASI = Wechsler Abbreviated Scale of Intelligence.

\begin{tabular}{ccccc}
\hline & $\begin{array}{c}\text { Borderline Personality } \\
\text { Disorder }(n=18)\end{array}$ & $\begin{array}{c}\text { Schizotypal Personality } \\
\text { Disorder }(n=18)\end{array}$ & $\begin{array}{c}\text { Healthy control } \\
(n=17)\end{array}$ & Statistic/p-value \\
\hline Age (years) & $36.8(11.7)$ & $33.6(9.6)$ & $31.4(7.7)$ & t-tests; all $p$-values $>0.12$ \\
Gender & $7 \mathrm{M} / 12 \mathrm{~W}$ & $11 \mathrm{M} / 7 \mathrm{~W}$ & $10 \mathrm{M} / 7 \mathrm{~W}$ & chi-square; all $p$-values $>0.14$ \\
Education (years) & $15.6(3.3)$ & $14.9(3.3)$ & $14.0(2.5)$ & t-tests; all $p$-values $>0.11$ \\
WASI & $104.9(19.0)$ & $104.8(9.9)$ & $108.2(18.1)$ & t-tests; all $p$-values $>0.50$ \\
\hline
\end{tabular}

months. Four of the SPD patients and 10 of the BPD patients had past major depressive disorder (MDD) occurring at least six months prior to entering the study.

As required for a diagnosis of SPD using the DSM-IV, SPD participants endorsed at least five of the nine SPD criteria with a rating greater than or equal to 1.0 [each DSM-IV criteria was rated on a 4-point scale (converted from the typical SIDP-IV scale of 0 to 3 ) and consisting of the following: $0=$ absent, $0.5=$ somewhat present, 1.0 = definitely present, 2.0 = severe], and were only included if they met no more than three BPD criteria with two items rated as 1.0 and one item rated as 0.5. BPD participants met at least five of the nine DSM-IV criteria for BPD and were allowed to endorse no more than three SPD criteria with two items rated as 1.0 and one item rated as 0.5. Healthy controls were allowed to meet for at least two of the nine BPD and SPD criteria, respectively. In our laboratory, the intraclass correlation for BPD diagnosis was 0.80 and for SPD it was 0.73 .

The majority of participants were recruited via advertisements in local newspapers. Approximately $10 \%$ of the SPD and BPD participants were recruited by referral from the outpatient psychiatric clinic at Icahn School of Medicine at Mount Sinai. The study was approved by the IRB and all participants provided written informed consent.

\subsection{Procedure}

Participants were initially administered the Wechsler Abbreviated Scale of Intelligence to ensure they were matched on general cognitive functioning across groups. They subsequently completed several of the attention/ executive functioning tasks from the Cambridge Neuropsychological Test Automatic Battery (CANTAB) and the Subjective Emotion Questionnaire to assess aspects of mood.

\subsection{Measures}

The Cambridge Neuropsychological Test Automated Battery. (CANTAB; CeNeS Ltd., Cambridge). The CANTAB is a sensitive and specific cognitive assessment tool with extensive validation (Robbins et al., 1998) and has demonstrated adequate reliability (Robbins et al., 1994). The specific CANTAB tasks were administered on a touch-screen computer.

All of the measures administered included the following:

Delayed Matching to Sample (DMS). This is a measure of short term visual recognition and match-to-sample abilities. The participant is presented with a complex visual pattern and then, following a delay (which varies in duration across the trials), must choose which one they had seen previously. Additional trials require the participant to select the correct stimulus while it is still in view. The outcome measure is the percentage of correct responses provided by the participant.

Rapid Visual Information Processing (RVP). This task is a test of sustained attention. A white box appears in the center of the computer screen and opens to reveal digits from two to nine that are presented in pseudo-random order at the rate of 100 digits per minute. The participant is required to detect consecutive odd or even sequences of digits. The outcome measure is a signal detection measure of error sensitivity (ranging from zero to one).

Reaction Time (RTI). This test measures visuo-motor processing speed. A yellow dot is displayed on the screen in one of five locations, and participant is required to hold down the press pad or touch the screen as soon as it appears. The outcome measure is the reaction time (in milliseconds). 
Spatial Span (SSP). The SSP task assesses working memory capacity. White squares are displayed, some of which change color in a varying sequence. The participant is asked to touch each of the boxes in the same order as they were colored by the computer, and the number of boxes in the sequence is increased from 2 to 9 at the end of the test. The outcome measure is "span length", i.e. the longest sequence successfully recalled by the subject.

Stockings of Cambridge (SOC). This is a measure of spatial planning and problem solving. The participant is presented with two displays containing three colored balls and required to use the balls in one of the displays to copy the pattern shown in the other display. The outcome measure is number of problems solved in the minimum number of moves possible.

The Wechsler Abbreviated Scale of Intelligence. (WASI; Psychological Corporation, 1999). The WASI is a 45-minute measure of general intellectual ability that includes four subtests and yields an estimate of the Full Scale IQ (Standard Score).

Subjective Emotion Questionnaire. (SEQ; Berlin \& Rolls, 2004; Berlin et al., 2004, 2005). Using a 4-point Likert scale, this questionnaire measures how often participants experience sadness, anger, fear, happiness, and disgust in their everyday lives. The outcome measure is the total of the five individual item ratings, with the happiness item rating reverse scored.

\subsection{Statistical Analyses}

A one-way analysis of variance (ANOVA) was performed on each variable to determine if mean scores differed significantly between the three groups. The Holm-Bonferroni method was applied to correct for multiple comparisons and then a protected Tukey's Honest Significant Difference (HSD) post-hoc test was performed to identify the specific source of the difference. Of note, Spatial Span, Spatial Working Memory, and Stockings of Cambridge data were not collected for one of the SPD participants; however, this individual was included in the other analyses.

Pearson correlations and Spearman-Rank Correlations (2-tailed), for non-normally distributed data, were performed within each group to investigate relationships among the neuropsychological variables and mood variables. Since DMS percent correct (all delays) was the only significant variable, it was correlated with mood variables and the alpha level was lowered to account for the number of comparisons performed (Bonferroni correction).

\section{Results}

\subsection{Neuropsychological Measures}

Performance on the following dependent variable significantly differed among the groups: Delayed Matching to Sample all delays (a delay of 0 , 4, or 12 seconds is introduced between the covering sample pattern and the choice patterns), $F(2,51)=5.06, p=0.009, \eta \rho^{2}=0.2$. Of note, results from the Spatial Span task only approached significance after correcting for multiple comparisons, $F(2,50)=3.04, p=0.057$ (trend level, $\eta \rho^{2}=$ 0.11). Results from the other CANTAB subtests were not significant (see Table 2).

As such, individuals with SPD performed significantly worse on the Delayed Matching to Sample task (all delays) than the healthy control participants. Specifically, compared with healthy controls, SPD participants correctly answered a significantly smaller percentage of Delayed Matching to Sample trials, in which the stimulus and matching answer choices were presented with varying delays, $p<0.01$, Tukey's HSD, Figure 1 . However, SPD and BPD participants did not differ from one another, and the BPD participants did not differ from healthy controls, $p$ 's $>0.01$.

\subsection{Mood Questionnaire}

With regard to findings from a mood inventory (i.e., the Subjective Emotion Questionnaire, sadness, anger, fear, happiness, and disgust), results revealed significant differences among groups on the five measures, $F(2,49)=$ $11.28, p<0.0001, F(2,49)=10.77, p=0.0001, F(2,49)=4.86, p=0.01, F(2,49)=8.05, p<0.001$, and $F(2$, 49) $=9.81, p=0.0002$, respectively.

In general, BPD participants experienced more subjective sadness, fear, and anger, and less happiness than HC and SPD participants; SPD participants also reported experiencing significantly less happiness than healthy 
Table 2. Neuropsychology data by diagnostic group.

\begin{tabular}{ccccc}
\hline & \multicolumn{3}{c}{ Mean score (SD) } & F, df, $p$ \\
\cline { 2 - 4 } & Borderline PD & Schizotypal PD & Healthy control & \\
Delayed matching to sample & & & \\
Percent correct, all delays & $85.6(8.39)$ & $82.9(12.73)^{*}$ & $92.6(4.17)$ & 5.06, (2, 51), $p<0.01$ \\
Reaction time & & & & \\
5-choice reaction time & $376.6(56.79)$ & $431.9(162.34)$ & $382.7(78.29)$ & $1.41,(2,51), n s$ \\
Rapid visual information processing & $0.9(0.06)$ & $0.9(0.06)$ & $0.9(0.06)$ & $1.52,(2,51), n s$ \\
Spatial span, span length & $6.1(1.24)$ & $5.6(1.28)$ & $6.7(1.45)$ & $3.04,(2,50), p=0.057$ \\
Stockings of Cambridge, Moves & $8.8(1.75)$ & $8.2(2.22)$ & $8.8(2.51)$ & $0.40,(2,50), n s$ \\
\hline
\end{tabular}

*Tukey's HSD post-hoc test, $p<0.01$, different from healthy controls; $\mathrm{SD}=$ standard deviation; $n s=$ not significant.

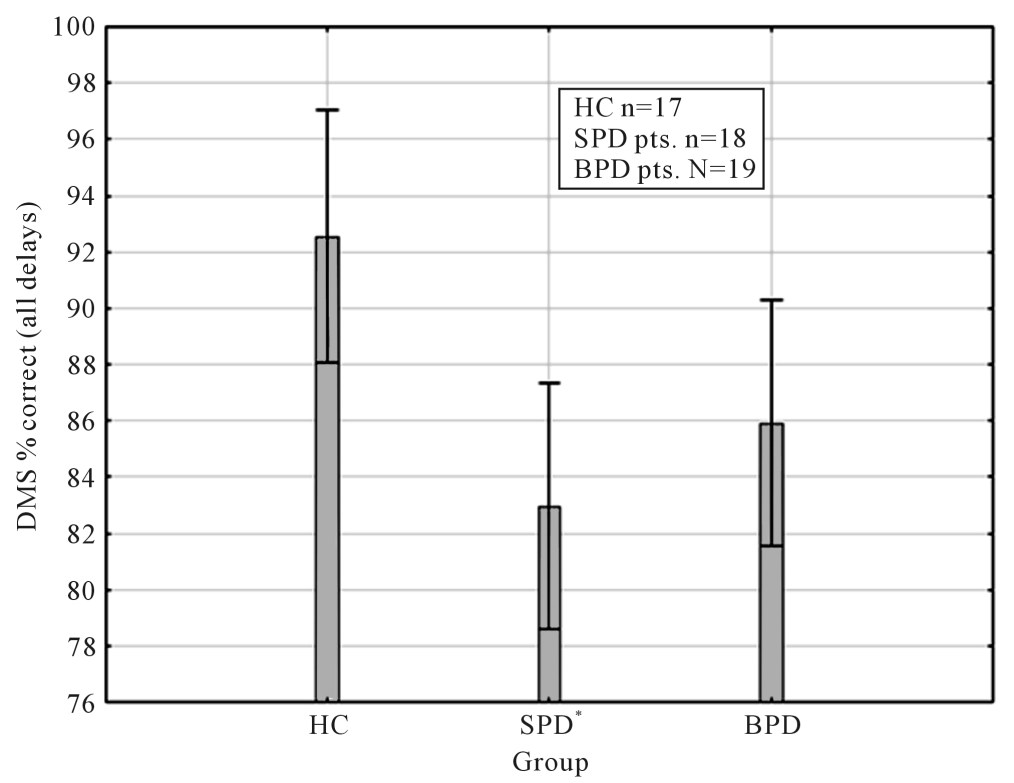

Figure 1. Mean percent correct on the Delayed Matching to Sample (DMS) Task for the healthy control (HC), borderline personality disorder (BPD), and schizotypal personality disorder (SPD) groups. Compared with the HC group, individuals with BPD and SPD correctly answered a lower percentage of trials in which the stimulus and matching answer choices were presented at varying delays, $F(2,51)=5.06, p<0.01$. Error bars represent the standard error of the mean. *post-hoc test indicated that the SPD group is significantly different from the HC group, $p<0.01$.

control participants. Specifically, BPD participants reported experiencing more sadness than SPD participants and HCs as reported on the subjective sadness variable, $p=0.008$ and $p=0.0002$ (Tukey's HSD), respectively. BPD participants reported experiencing more anger based on the subjective anger variable than both HC and SPD participants, $p=0.0003$ and $p=0.002$ (Tukey's HSD), respectively. BPD participants also indicated that they were experiencing more subjective fear $(p=0.009)$ and less happiness $(p=0.003)$ than HC participants (Tukey's HSD). Additionally, SPD participants reported that they were having significantly less happiness than healthy controls, $p=0.003$, Tukey's HSD.

Of note, no significant relationships were detected between DMS performance and mood for SPD participants and healthy controls, respectively. Therefore, mood was not included as a control variable within the aforementioned analyses. 


\section{Discussion}

This study aimed to examine aspects of attention/executive functioning and mood in individuals with BPD and SPD, respectively. Consistent with our group’s previous results suggesting relative weakness with spatial working memory in SPD individuals as compared with healthy controls, current findings once again revealed SPD relative difficulty with short-term visual memory compared with that of HC. These results are also consistent with prior SPD reports of weakness in aspects of short-term memory (Mitropoulou et al., 2005; Voglmaier et al., 2000).

All of that said, the extent to which SPD individuals appear to be experiencing primary difficulties with short-term memory abilities remains unclear. For example, the possibility that such weakness is secondary, at least in part, to attention problems cannot be ruled out, especially given findings that simple attention span in SPD patients trended towards significance, as compared with HC. More specifically, it is possible that variable attention skills in individuals with SPD may, indeed, impact their performance on short-term memory tasks. Additionally, the possibility that the SPD-HC related differences are attributable to other aspects of attention/executive functioning, such as problems with the executive control processes of memory (e.g., encoding, organization, discriminability) should also be considered. Still, due to prior work indicating that resource/processing capacity limitations constitute a primary weakness in SPD (Harvey, Reichenberg, Romero, Granholm, \& Siever, 2006), it is also quite possible that this limitation remains the primary reason for the current study's findings.

It is important to address the present study's failure to detect any BPD-HC differences, particularly with regard to the hypothesized area of concern in individuals with BPD, i.e. planning/inhibition skills, given prior work implicating this domain of functioning in BPD. With regard to discrepant results in performance of planning measures, it is helpful to note that the current study used a different version of the Tower of London task typically used in studies that have reported weaknesses. Thus, it is certainly possible that failure to detect group differences may be due to measure-related factors. Further, in general, considerable variability has been observed in the literature assessing neuropsychological functioning in individuals with BPD. In this respect, some research has argued that such variability could be attributable to the impact of situational and/or emotional factors on BPD neuropsychological performance (e.g., Domes, Winter, Schnell, Vohs, Fast, \& Herpertz, 2006). While the current study did not detect a significant relationship between neuropsychological performance and mood, it is possible that the measure used to assess mood (i.e., SEQ) was not sensitive enough to pick up on significant mood factors at the time of participation in the study, as it primarily serves as a measure of mood traits rather than current mood state.

There are a number of additional limitations that may also help account for the lack of support for prior work. The sample size was relatively small and selective which limits the generalizability and power of the study. Thus, some important group differences may not have been elucidated. As mentioned above, several BPD and SPD participants met criteria for past MDD, which further supports the argument that mood-related factors may have confounded results. Although patients with a past history of MDD were required to be in full remission for six months prior to the present study, we cannot rule out the possibility that mood may have continued to impact functioning, especially given the longstanding/residual nature of severe mood problems in general. Also, this same exclusionary condition (no past history of MDD, BPD patient could not meet for more than three SPD symptom criteria and vice/versa) may have also limited our findings given arguments that a "true" personality disorder sample would exhibit such symptoms, as well as other Axis I/II difficulties. Of note, while some might pose the same argument regarding the fact that our patients were unmedicated at the time of the study (and thus, not fully representative of a personality-disorder sample), we did remove the potential confound of medication on task performance.

\section{Conclusion}

Overall, findings from the current study reveal patterns of fronto-subcortical weaknesses in two different personality disorders, respectively. However, the BPD results revealed mood-related fronto-subcortical difficulties, whereas the SPD results primarily implicated fronto-subcortical disruption of short-term memory abilities. That is, while the BPD group did not exhibit difficulty on any of the cognitive measures as compared with HCs, they did endorse significantly higher levels of affective disturbance relative to HCs and the individuals with SPD. In this respect, it is crucial for future research to continue investigating the different cognitive and emotional factors that may or may not be contributing to functional difficulties in BPD and personality disorder populations in 
general. Such work will not only enable practitioners to target key weaknesses within the therapeutic context (e.g., instruct patients on how to practice active listening on a regular basis, train patients to use organizational devices and review the information contained within the device as often as possible), it may also help facilitate diagnostic and construct validity and improve assessment practices.

\section{Acknowledgements}

This work was supported by NIMH grant R01MH073911 to Dr. Hazlett. Partial support also came from CTSA grant UL1TR000067 from the National Center for Advancing Translational Sciences (NCATS), a component of the National Institutes of Health (NIH). We also appreciate support from the Mental Illness Research, Education, and Clinical Center (VISN 3 MIRECC) at the James J. Peters VA Medical Center, Bronx, NY.

\section{Conflict of Interest}

None of the authors have any conflicts of interest.

\section{References}

Berlin, H. A, Rolls, E. T., \& Kischka, U. (2004). Impulsivity, Time Perception, Emotion and Reinforcement Sensitivity in Patients with OFC Lesions. Brain, 127, 1108-1126. http://dx.doi.org/10.1093/brain/awh135

Berlin, H. A., \& Rolls, E. T. (2004). Time Perception, Impulsivity, Emotionality, and Personality in Self-Harming Borderline Personality Disorder Patients. Journal of Personality Disorders, 18, 358-378. http://dx.doi.org/10.1521/pedi.2004.18.4.358

Berlin, H. A., Rolls, E. T., \& Iversen, S. D. (2005). Borderline Personality Disorder, Impulsivity, and the Orbitofrontal Cortex. American Journal of Psychiatry, 162, 2360-2373. http://dx.doi.org/10.1176/appi.ajp.162.12.2360

Dell’Osso, B., Berlin, H. A., Serati, M., \& Altamura, A. C. (2010). Neuropsychobiological Aspects, Comorbidity Patterns and Dimensional Models in Borderline Personality Disorder. Neuropsychobiology, 61, 169-179. http://dx.doi.org/10.1159/000297734

Domes, G., Winter, B., Schnell, K., Vohs, K., Fast, K., \& Herpertz, S. C. (2006). The Influence of Emotions on Inhibitory Functioning in Borderline Personality Disorder. Psychological Medicine, 36, 1163-1172. http://dx.doi.org/10.1017/S0033291706007756

First, M. B., Spitzer, R., Gibbon, M., \& Williams, J. (1996). Structured Clinical Interview for Axis I Disorders-Patient Edition. New York: Biometrics Research, New York State Psychiatric Institute.

Goldstein, K. E., Hazlett, E. A., Savage, K. R., Berlin, H. A., Hamilton, H. K., Zelmanova, Y., Look, A. E., Koenigsberg, H. W., Mitsis, E. M., Tang, C. Y., McNamara, M., Siever, L. J., Cohen, B. H., \& New, A. S. (2011). Dorso- and VentroLateral Prefrontal Volume and Spatial Working Memory in Schizotypal Personality Disorder. Behavioral Brain Research, 218, 335-340. http://dx.doi.org/10.1016/j.bbr.2010.11.042

Harvey, P. D., Reichenberg, A., Romero, M., Granholm, E., \& Siever, L. J. (2006). Dual-Task Information Processing in Schizotypal Personality Disorder: Evidence of Impaired Processing Capacity. Neuropsychology, 20, 453-460. http://dx.doi.org/10.1037/0894-4105.20.4.453

Kavoussi, R. J., \& Siever, L. J. (1992). Overlap between Borderline and Schizotypal Personality Disorders. Comprehensive Psychiatry, 33, 7-12. http://dx.doi.org/10.1016/0010-440X(92)90072-X

LeGris, J., \& Van Reekum, R. (2006). The Neuropsychological Correlates of Borderline Personality Disorder and Suicidal Behavior. The Canadian Journal of Psychiatry, 51, 131-142.

Lenzenweger, M. S., Lane, M. C., Loranger, A. W., \& Kessler, R. C. (2007). DSM-IV Personality Disorders in the National Comorbidity Survey Replication. Biological Psychiatry, 62, 553-564. http://dx.doi.org/10.1016/j.biopsych.2006.09.019

McCloskey, M. S., Phan, K. L., \& Coccaro, E. F. (2005). Neuroimaging and Personality Disorders. Current Psychiatry Reports, 7, 65-72. http://dx.doi.org/10.1007/s11920-005-0027-2

McGlashan, T. H. (1987). Testing DSM-III Symptom Criteria for Schizotypal and Borderline Personality Disorders. Archives of General Psychiatry, 44, 143-148. http://dx.doi.org/10.1001/archpsyc.1987.01800140045007

Mitropoulou, V., Harvey, P. D., Maldari, L. A., Moriarty, P. J., New, A. S., Silverman, J. M., et al. (2002). Neuropsychological Performance in Schizotypal Personality Disorder: Evidence Regarding Diagnostic Specificity. Biological Psychiatry, 52, 1175-1182. http://dx.doi.org/10.1016/S0006-3223(02)01426-9

Mitropoulou, V., Harvey, P. D., Zegarelli, G., New, A. S., Silverman, J. M., \& Siever, L. J. (2005). Neuropsychological Performance in Schizotypal Personality Disorder: Importance of Working Memory. The American Journal of Psychiatry, 162, 
1896-1903. http://dx.doi.org/10.1176/appi.ajp.162.10.1896

Pfohl, B., Blum, N., \& Zimmerman, M. (1997). Structured Clinical Interview for DSM-IV Personality (SIDP-IV). Washington DC: American Psychiatric Press.

Psychological Corporation (1999). Wechsler Abbreviated Scale of Intelligence (WASI) Manual. San Antonio, TX: Psychological Corporation.

Robbins, T. W., James, M., Owen, A. M., Sahakian, B. J., Lawrence, A. D., McInnes, L., \& Rabbit, P. M. (1998). A Study of Performance on Tests from the CANTAB Battery Sensitive to Frontal Lobe Dysfunction in a Large Sample of Normal Volunteers: Implications for Theories of Executive Functioning and Cognitive Aging. Cambridge Neuropsychological Test Automated Battery. Journal of International Neuropsychological Society, 4, 474-490. http://dx.doi.org/10.1017/s1355617798455073

Robbins, T. W., James, M., Owen, A. M., Sahakian, B. J., McInnes, L., \& Rabbitt, P. (1994). Cambridge Neuropsychological Test Automated Battery (CANTAB): A Factor Analytic Study of a Large Sample of Normal Elderly Volunteers. Dementia and Geriatric Cognitive Disorders, 5, 266-281. http://dx.doi.org/10.1159/000106735

Ruocco, A. C. (2005). The Neuropsychology of Borderline Personality Disorder. Psychiatry Research, 137, 191-202. http://dx.doi.org/10.1016/j.psychres.2005.07.004

Seres, I., Unoka, Z., Nikoletta, B., Áspán, N., \& Keri, S. (2009). The Neuropsychology of Borderline Personality Disorder: Relationship with Clinical Dimensions and Comparison with Other Personality Disorders. Journal of Personality Disorder, 23, 555-562. http://dx.doi.org/10.1521/pedi.2009.23.6.555

Voglmaier, M. M., Seidman, L. J., Niznikiewicz, M. A., Dickey, C. C., Shenton, M. E., \& McClarley, R. W. (2000). Verbal and Nonverbal Neuropsychological Test Performance in Subjects with Schizotypal Personality Disorder. The American Journal of Psychiatry, 157, 787-793. http://dx.doi.org/10.1176/appi.ajp.157.5.787 